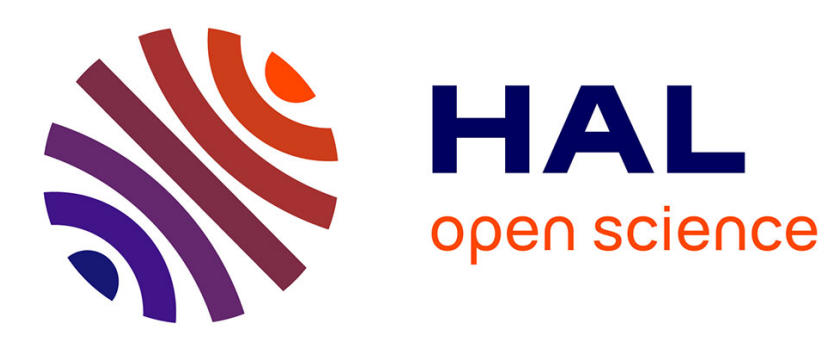

\title{
Probabilistic Modeling of Brittle Fracture Including 3-D Effects on Constraint Loss and Ductile Tearing
}

\author{
C. Ruggieri, R. Dodds
}

\section{To cite this version:}

C. Ruggieri, R. Dodds. Probabilistic Modeling of Brittle Fracture Including 3-D Effects on Constraint Loss and Ductile Tearing. Journal de Physique IV Proceedings, 1996, 06 (C6), pp.C6-353-C6-362. 10.1051/jp4:1996635 . jpa-00254463

\section{HAL Id: jpa-00254463 https://hal.science/jpa-00254463}

Submitted on 1 Jan 1996

HAL is a multi-disciplinary open access archive for the deposit and dissemination of scientific research documents, whether they are published or not. The documents may come from teaching and research institutions in France or abroad, or from public or private research centers.
L'archive ouverte pluridisciplinaire HAL, est destinée au dépôt et à la diffusion de documents scientifiques de niveau recherche, publiés ou non, émanant des établissements d'enseignement et de recherche français ou étrangers, des laboratoires publics ou privés. 


\title{
Probabilistic Modeling of Brittle Fracture Including 3-D Effects on Constraint Loss and Ductile Tearing
}

\author{
C. Ruggieri and R.H. Dodds Jr. \\ Department of Civil Engineering, University of Illinois at Urbana-Champaign, Urbana, Illinois 61801, \\ U.S.A.
}

\begin{abstract}
This study presents a probabilistic, 3-D framework to describe brittle fracture in structural components which incorporates weakest link statistics and a micromechanics model reflecting local damage of material. The Weibull stress $\left(\sigma_{w}\right)$ emerges as a fracture parameter to define conditions leading to local material failure. This parameter is correlated with the macroscopic loading and used to describe overall fracture conditions in a cracked solid exhibiting both loss of constraint and stable crack growth prior to cleavage fracture. The paper also describes an application of the proposed framework to predict the measured geometry and ductile tearing effects on the statistical distribution of cleavage fracture toughness for an HSLA steel.
\end{abstract}

\section{INTRODUCTION}

A fundamental concept underlying fracture mechanics in both linear-elastic and elasto-plastic regimes relies on the correlation of unstable crack propagation in different cracked bodies based on the similarity of their respective near-tip stress and strain fields. Conventional fracture assessments of large engineering structures using laboratory specimen data most often employ a one-parameter characterization of loading and toughness $\left(K_{\mathrm{I}}, J\right.$ or CTOD). The more recent two-parameter approaches ( $T$-stress and $J-Q$ methodologies) retain contact with traditional fracture mechanics and provide a concise framework to represent measured toughness values in terms of a $J-T$ or $J-Q$ locus. However, they do not provide a means to predict the effects of constraint variations and prior ductile tearing on toughness. Such predictions necessarily require a more detailed description of the fracture process, and are most often accomplished through recourse to micromechanics models that employ local fracture criteria (collectively referred to as local approaches). Further, the random inhomogeneity in local features of the material generally causes large scatter in measured fracture toughness values $\left(K_{\mathrm{I} c}, J_{c}\right.$ or CTOD). Consequently, realistic methodologies for fracture assessments of engineering components must adopt a probabilistic, rather than a deterministic, treatment of fracure.

In this study, we describe a computational framework for brittle fracture which incorporates weakest link statistics and a micromechanics model reflecting local damage of the material. The approach addresses the strongly interacting effects of ductile tearing and constraint variations on (macroscopic) cleavage fracture toughness most apparent in the ductile-to-brittle transition (DBT) region. The Weibull stress $\left(\sigma_{w}\right)$ [1] emerges as a probabilistic fracture parameter to define conditions leading to (local) material failure. Unstable crack propagation occurs at a critical value of $\sigma_{w}$ which may be attained prior to or following some amount of stable, ductile crack extension. When implemented in a finite element code, the computational model predicts the evolution of Weibull stress with applied load (conveniently measured by $J$ in the present work) while the crack tip undergoes first blunting and then stable, ductile crack extension. Here, a central feature of our framework incorporates realistic modeling of ductile crack growth using the computational cell methodology to define the evolution of near-tip stress fields during crack extension. A simplified form of the Gurson-Tvergaard (GT) $[2,3]$ constitutive model for dilatant plasticity serves to predict microscopic void growth within a layer of cells defined over the crack plane. The Weibull stress then describes both the effects of stressed volume and the potential- 
ly strong changes in the character of the near-tip stress fields due to constraint loss and ductile crack extension.

The numerical results focus on application of the micromechanics model to predict specimen geometry and stable crack growth effects on the macroscopic fracture toughness $\left(J_{c}\right)$ in an HSLA steel. Fracture toughness values measured experimentally for a high-constraint geometry that exhibit no prior ductile tearing are effectively transferred to a different geometry having much lower constraint and in which tearing precedes cleavage. The inherent difficulty in predicting the scatter of experimental fracture toughness, as well as constraint and ductile tearing effects, within the scope of conventional procedures appears greatly reduced in the methodology presented here.

\section{PROBABILISTIC FRAMEWORK FOR CLEAVAGE FRACTURE}

\subsection{Probability Distribution for the Fracture Stress of Cracked Solids}

Extensive work on cleavage fracture in ferritic steels demonstrates that cracking of grain boundary carbides in the course of plastic deformation and subsequent extension of these cracks into the surrounding matrix governs failure. This type of fracture is a highly localized phenomenon which exhibits strong sensitivity to material characteristics at the microlevel. Consequently, the random nature of cleavage fracture drives the development of a relationship to couple macroscopic fracture behavior with microscale events. Existing probabilistic models generally adopt weakest link arguments to yield asymptotic distributions for the fracture strength of brittle materials (see, e.g., [1, 4-10]). We consider the probability distribution for the fracture stress of a cracked solid defined by a two-parameter Weibull distribution [11] in the form

$$
\mathscr{P}\left(\sigma_{1}\right)=1-\exp \left[-\frac{1}{V_{0}} \int_{\Omega}\left(\frac{\sigma_{1}}{\sigma_{u}}\right)^{m} d \Omega\right]=1-\exp \left[-\left(\frac{\sigma_{w}}{\sigma_{u}}\right)^{m}\right]
$$

where $\Omega$ denotes the volume of the (near-tip) fracture process zone and $\sigma_{1}$ is the maximum principal stress acting on material points inside the fracture process zone. Parameters $m$ and $\sigma_{u}$ appearing in Eq. (1) denote the Weibull modulus and the scale parameter of the Weibull distribution. Following Beremin [1], the Weibull stress is defined as the stress integral

$$
\sigma_{w}=\left[\frac{1}{V_{0}} \int_{\Omega} \sigma_{1}^{m} d \Omega\right]^{1 / m}
$$

Equation (1) implicitly defines a zero threshold stress for fracture; consequently, stresses vanishingly small compared to the fracture stress yield a non-zero (albeit small) probability for fracture. A more refined form of the limiting distribution for the fracture stress of a cracked solid using a threshold stress, $\sigma_{t h}$, has been explored by Bakker and Koers [8] (B\&K) and Ruggieri and Dodds [10] (R\&D). Here, $\sigma_{t h}$ has the physical interpretation of a lower-bound strength for fracture; the failure probability for the cracked solid becomes zero for any stress below $\sigma_{t h}$. However, R\&D [10] show that such refinement does not appear to provide significant improvements in the fracture behavior predicted by the present methodology.

\subsection{Inclusion of Ductile Tearing Effects Prior to Cleavage Fracture}

The Weibull stress describes local conditions leading to unstable (cleavage) failure and appears, at least as a first approximation, to remain applicable during small amounts of ductile crack extension. However, the potential fracture process zone for an extending crack now comprises three distinct zones depending upon the material's stress history. Figure 1 provides a schematic illustration of the three zones, denoted as $A, B$, and $C$. Material in the unloaded zone $A$ behind the current physical location of the advancing crack tip, but previously located ahead of the tip, experienced severe stress and strain fields without triggering cleavage fracture. Due to blunting at the location of the physical crack tip, the peak value of opening mode stress develops at a small distance (roughly the blunted opening) ahead of the tip. Material in the region between the crack tip and the peak stress location (zone $B$ ) experiences significant damage due to void growth with a corresponding stress reduction. Earlier in the loading history, this material (which still contains potential cleavage sites) was ahead of the peak stress location, and 
thus has experienced unloading in terms of the continuum stress field accompanied by a large increase in (tensile) plastic strain. Finally, material located outside this blunting region (zone $C$ ) experiences increased stresses with consequent generation of new microcracks and cracked carbides due to progressive plastic deformation.

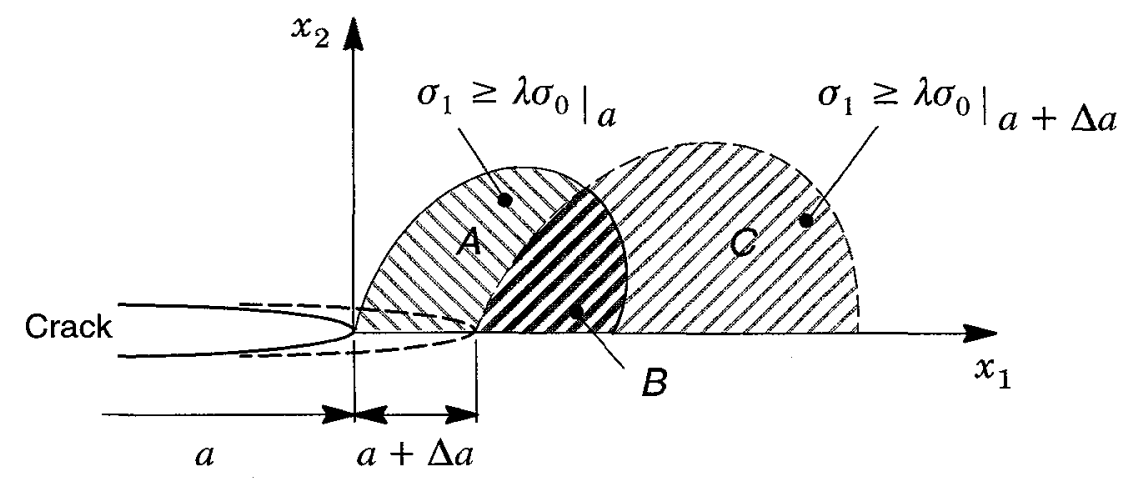

Figure 1 Evolution of the fracture process zone for a growing crack.

The evolution of Weibull stress during crack growth obviously depends on the choice of the fracture process zone. Cleavage cracking in the unloaded region behind the current crack tip (zone $A$ ) does not appear sufficient to trigger unstable propagation of the macroscopic crack. Furthermore, it seems not at all certain that material in zone $A$, which has previously suffered some damage due to growth, effectively contributes to the current failure probability of the cracked body.

In contrast, small amounts of ductile crack growth can modify the stress history of material ahead of crack tip, especially in zone $C$, which affects strongly the propensity for unstable propagation of the macroscopic crack. For material in zones $B$ and $C$, previous studies of ductile crack growth [12,13] have revealed that: (1) for geometries with low constraint prior to growth, stress triaxiality ahead of the crack tip increases significantly during growth due to elastic unloading behind and macroscopic re-sharpening of the advancing tip, (2) for geometries with high constraint prior to growth, only minor elevations in peak stress triaxiality occur, and (3) for all cases, the extent of the region of higher stress triaxiality increases leading to a larger process zone for cleavage fracture. Thus, we propose to define the active fracture process zone, which moves forward with the advancing tip and increases in size, as the loci $\sigma_{1} \geq \lambda \sigma_{0}$, encompassing zones $B$ and $C$, with $\lambda \approx 2$. As illustrated in Fig. 1 , the development of such a region is given by a snapshot of the stress field ahead of the growing crack; points on such a contour all lie within the forward sector $|\theta| \leq \pi / 2$. Consequently, the $3-D$ form of the Weibull stress for a growing crack becomes simply

$$
\sigma_{w}=\left[\frac{1}{V_{0}} \int_{\Omega^{*}} \sigma_{1}{ }^{m} d \Omega^{*}\right]^{1 / m}
$$

where $\Omega^{*}$ denotes the volume of the active fracture process zone, $\sigma_{1} \geq \lambda \sigma_{0}$, which moves forward with the advancing tip. The proposed generalization of $\sigma_{w}$ to include ductile tearing maintains the relative simplicity of computations while, at the same time, fully incorporating the effects of alterations in the stress field ahead of the crack tip.

In related work to characterize cleavage fracture in the DBT transition region for ferritic steels, $B \& K$ [8] also extend the Weibull stress concept to include damage of material located within the region where partial unloading occurs (zone $B$ ). However, they replace the current stress in Eq. (3) with the maximum stress that the material point has experienced during the entire loading history. Compared to the present proposal, their procedure may emphasize the contribution of material in zone $B$ toward the total failure probability. Section 4.2 addresses this issue and compares the evolution of $\sigma_{w}$ with macroscopic loading (as characterized by $J$ ) using the present definition of the active process zone and the maximum stress approach. 


\subsection{Finite Element Representation of the Weibull Stress}

In isoparametric space, the current (deformed) Cartesian coordinates $x_{i}$ of any point inside a 8-node tri-linear element are related to the parametric coordinates $\eta_{i}$ using the shape functions corresponding to the $k$-th node [14]. Let $|J|$ denote the determinant of the standard coordinate Jacobian between deformed Cartesian and parametric coordinates. Then using standard procedures for integration over element volumes, the Weibull stress has the form

$$
\sigma_{w}=\left[\frac{1}{V_{0}} \sum_{n_{e}} \int_{\Omega_{e}} \sigma^{m} d \Omega_{e}\right]^{1 / m}=\left[\frac{1}{V_{0}} \sum_{n_{e}} \int_{-1}^{1} \int_{-1}^{1} \int_{-1}^{1} \sigma_{1}^{m}|J| d \eta_{1} d \eta_{2} d \eta_{3}\right]^{1 / m}
$$

where $n_{e}$ is the number of elements inside the fracture process zone near the crack tip and $\Omega_{e}$ is the volume of the element. The process zone used here includes all material inside the loci $\sigma_{1} \geq \lambda \sigma_{0}$, with $\lambda=2$. For computational simplicity, an element is included in the fracture process zone if the $\sigma_{1}$ computed at $\eta_{1}=\eta_{2}=\eta_{3}=0$ exceeds $2 \sigma_{0}$. This expression for the Weibull stress represents the integral form in parametric space of Beremin's formulation [1].

\section{NUMERICAL PROCEDURES AND COMPUTATIONAL MODELS}

\subsection{Ductile Crack Growth Using Computational Cells}

The computational cell methodology proposed by Xia and Shih [18-20] (X\&S) provides a model for ductile crack extension that includes a realistic void growth mechanism, and a microstructural lengthscale physically coupled to the size of the fracture process zone. Void growth remains confined to a layer of material symmetrically located about the crack plane, as illustrated in Fig. 2(a), and having thickness $D$, where $D$ is associated with the mean spacing of the larger, void initiating inclusions. This layer consists of cubical cell elements with dimension $D$ on each side; each cell contains a cavity of initial volume fraction $f_{0}$ (the initial void volume divided by cell volume). As a further simplification, the void nucleates from an inclusion of relative size $f_{0}$ immediately upon loading. Progressive void growth and subsequent macroscopic material softening in each cell are described with the Gurson-Tvergaard (GT) constitutive model for dilatant plasticity $[2,3]$. When $f$ in the cell incident on the current crack tip reaches a critical value, $f_{E}$, the computational procedures remove the cell thereby advancing the crack tip in discrete increments of the cell size. Figure 2(b) shows the typical, plane strain finite element representation of the computational cell model where symmetry about the crack plane requires elements of size $D / 2$. Material outside the computational cells, the "background" material, follows a conventional $J_{2}$ flow theory of plasticity and remains undamaged by void growth in the cells.

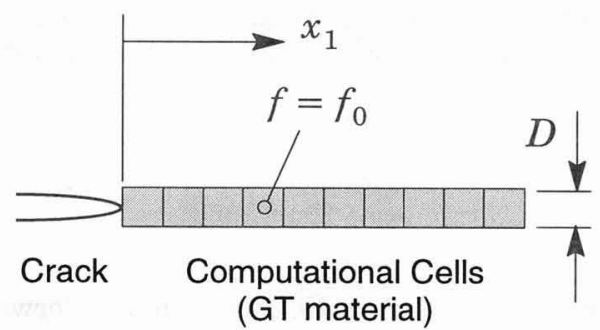

(a)

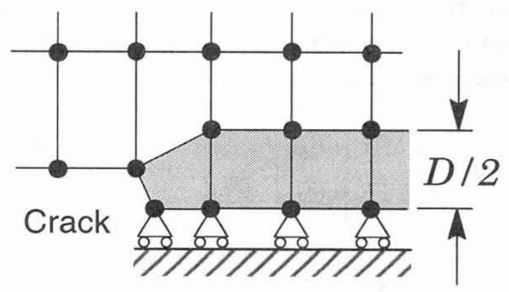

(b)

Figure 2 Modeling of ductile tearing using computational cells.

Material properties required for this methodology include: for the background material Young's modulus $(E)$, Poisson's ratio $(v)$, yield stress $\left(\sigma_{0}\right)$ and hardening exponent $(n)$ or the actual measured stress-strain curve; and for the computational cells: $D$ and $f_{0}$ (and of much less significance $f_{E}$ ). The background material and the matrix material of the cells generally have identical flow properties. Using an experimental $J-\Delta a$ curve obtained from a conventional SE(B) or C(T) specimen, a series of finite element analyses of the specimen are conducted to calibrate values for the cell parameters $D$ and $f_{0}$ 
which bring the predicted $J-\Delta a$ curve into agreement with experiment. The CTOD at initiation of ductile tearing provides a good starting value for $D$, with $f_{0}$ then varied to obtain agreement with the experiment. Alternatively, metallurgical surveys of inclusion volume fractions and sizes may be used with various packing arrangements (e.g. nearest neighbor distance) to estimate $D$ and/or $f_{0}$. Experience with plane-strain finite element analyses of $\mathrm{SE}(\mathrm{B})$ and $\mathrm{C}(\mathrm{T})$ specimens to estimate $D$ and $f_{0}$ for common structural and pressure vessel steels suggests values of $50-200 \mu \mathrm{m}$ for $D, 0.001-0.005$ for $f_{0}$, with $f_{E}$ typically $0.15-0.20$. Once determined in this manner using a specific experimental $R$-curve, $D$ and $f_{0}$ become "material" parameters and remain fixed in analyses of all other specimen geometries for the same material.

\subsection{Constitutive Models}

To describe the evolution of void growth and associated macroscopic material softening in the computational cells, we adopt the Gurson [2] and Tvergaard [3] potential function ( $g$ ) for plastic flow in porous media

$$
g\left(\sigma_{e}, \sigma_{m}, \bar{\sigma}, f\right)=\left(\frac{\sigma_{e}}{\bar{\sigma}}\right)^{2}+2 q_{1} f \cosh \left(\frac{3 q_{2} \sigma_{m}}{2 \bar{\sigma}}\right)-\left(1+q_{3} f^{2}\right)=0
$$

where $\sigma_{e}$ denotes the effective Mises (macroscopic) stress, $\sigma_{m}$ is the mean (macroscopic) stress, $\bar{\sigma}$ is the current flow stress of the cell matrix material and $f$ defines the current void fraction. Under multiaxial stress states, $\sigma_{e}=\left(3 S_{i j} S_{i j} / 2\right)^{1 / 2}$ where $S_{i j}$ denotes the deviatoric components of Cauchy stress. Factors $q_{1}, q_{2}$ and $q_{3}$ introduced by Tvergaard improve the model predictions for periodic arrays of cylindrical and spherical voids; here we use $q_{1}=1.25, q_{2}=1.0$ and $q_{3}=q_{1}^{2}$.

An additional contribution to the void growth rate arises from the nucleation of new voids caused by large plastic strains at much stronger, smaller inclusions dispersed into the matrix. The volume fraction of voids increases over an increment of load due to continued growth of existing voids and due to the formation of new voids caused by interfacial decohesion of inclusions or second phase particles. Thus,

$$
d f=(1-f) d \epsilon_{k k}^{p}+\mathcal{A}\left(\bar{\epsilon}^{p}\right) d \bar{\epsilon}^{p}=(1-f) d \epsilon_{k k}^{p}+\frac{f_{N}}{s_{N} \sqrt{2 \pi}} \exp \left[-\frac{1}{2}\left(\frac{\bar{\epsilon}^{p}-\epsilon_{N}}{s_{N}}\right)^{2}\right] d \bar{\epsilon}^{p}
$$

where $\bar{\epsilon}_{p}$ denotes the matrix plastic strain and $\epsilon^{p}$ is the volumetric (macroscopic) plastic strain. Here, the nucleation component follows a normally distributed evolution model [15] with parameter $\mathcal{A}$ defined by the mean value, $\epsilon_{N}$, the standard deviation, $s_{N}$ and the volume fraction of void nucleating particles, $f_{N}$.

Because the GT constitutive model does not predict a realistic loss of macroscopic stress in a cell at large void fractions, e.g. $f>0.15$, we utilize an element extinction procedure (proposed by Tvergaard) to delete a cell from the model thereby advancing the crack. When the averaged value of $f$ at the Gauss points in a cell element reaches $f_{E}$, the cell stiffness is made zero and the remaining stresses are relaxed to zero following a linear traction-separation model (here we reduce the stresses linearly to zero over an additional 10\% elongation of the cell normal to the crack plane). Effects of the specific extinction process diminish quickly when the number of load increments used in an analysis limits the number of cells undergoing extinction to just one or two. Based upon previous analysis by X\&S [18], $f_{E}$ is here assigned the value of $0.15 \sim 0.20$.

The background material outside of the computational cells follows a $J_{2}$ flow theory with the Mises plastic potential obtained by setting $f \equiv 0$ in Eq. (5). The true stress-logarithmic strain behavior for the high-strength, low hardening material of the SE(B) specimens that were tested in the experimental program is modeled with a piecewise linear approximation to the measured response. Mechanical tensile tests, conducted on a standard longitudinal tensile specimen, provide the room temperature $\left(20^{\circ} \mathrm{C}\right)$ stress-strain data $[10,24]$ used in the numerical analyses.

\subsection{Finite Element Models}

Finite element analyses are conducted on conventional plane-sided SE(B) specimens $W / S=4$ with $a / W=0.1$ and $a / W=0.5$ ( $W$ is the width and $S$ is the span for the bend specimens). The geometry, size and material flow properties match those for specimens tested in the experiments described in Section 4. Figure 3 shows the 3-D finite element models for the specimens. Symmetry conditions permit model- 
ing of only one-quarter of the specimen with appropriate constraints imposed on the remaining ligament. Stationary and growing crack analyses for these specimens generate detailed descriptions of the near-tip stress fields used to construct the proposed toughness scaling model.

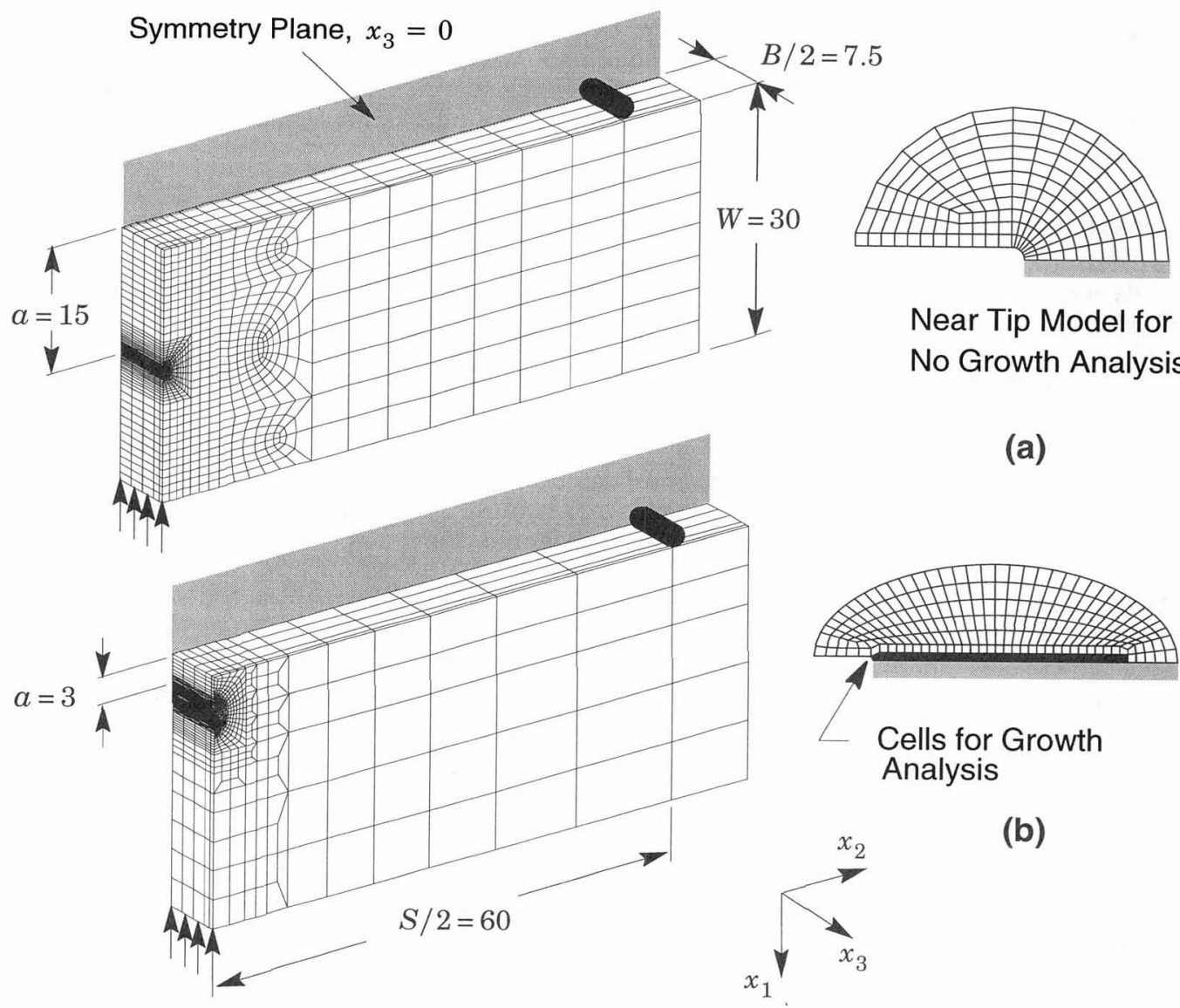

Figure 3 Finite element models for $3 \mathrm{P} \mathrm{SE}$ (B) specimens; (a) $a / W=0.5$; (b) $a / W=0.1$ (all units in mm).

Testing of the deeply notched bend specimens yielded fracture toughness values exhibiting a welldefined cleavage mode with no stable crack growth. For these specimens, a conventional mesh configuration having a focused ring of elements surrounding the crack front is used with a small key-hole at the crack tip; the radius of the key-hole is $25 \mu \mathrm{m}$. The mesh has 5000 elements arranged into 5 variable thickness, planar layers with sufficient refinement near the tip to provide adequate resolution of the stress fields. The thickest layer is defined at $x_{3}=0$ with thinner layers defined near the free surface $\left(x_{3}=B / 2\right)$ to accommodate strong $x_{3}$ variations in the stress distribution. For the shallow notched specimens, ductile tearing prior to cleavage fracture is observed in the experiments and requires a mesh with computational cells ahead of crack front. This 3-D model is constructed using 3000 elements arranged into 5 layers as in the model for the deep-crack specimen. Within each layer, 30 computational cells are defined along the crack ligament (fixed size of $D / 2 \times D / 2$ with $D / 2=100 \mu \mathrm{m}$ ) to model ductile tearing. Because the size of the computational cells used in growth analyses is dictated by the calibration procedure, it may not provide sufficient resolution of near-tip stress fields early in the loading history and prior to ductile tearing. Thus, a finite element analysis of a stationary crack with a key-hole having $25 \mu \mathrm{m}$ of radius at the crack tip (to maintain near-tip refinement similar to the model for the deep-crack specimen) is also conducted for the shallow notched specimen. This mesh contains 4000 elements with the same configuration as other models along the thickness (5 layers over $B / 2$ ). 


\section{PREDICTION OF $a / W$ AND $\triangle a$ EFFECTS ON TOUGHNESS}

\subsection{Experimental Toughness Data}

Toyoda et al. [24] conducted CTOD fracture mechanics tests on $\mathrm{SE}(\mathrm{B})$ specimens (plane-sided) with a fixed overall geometry and varying crack length to width ratios, $a / W$. The specimens have a loading span, $S=120 \mathrm{~mm}$, a width, $W=30 \mathrm{~mm}$, and thickness $B=15 \mathrm{~mm}$ (refer to Fig. 3 ). The material is quenched and tempered (QT), HSLA steel (663 MPa yield stress) with relatively low strain hardening $\left(\sigma_{u} / \sigma_{y s}=1.08\right)$ and very low $\mathrm{S}$ content. Exceptionally high tearing resistance and low transition temperature may thus be anticipated. In the present work, we limit attention to experimental data obtained for a deep crack $(a / W=0.5)$ and a shallow crack $(a / W=0.1)$ configuration. Testing of these configurations was carried out at $-120^{\circ} \mathrm{C}$ which corresponds to near lower-shelf behavior for the material with $a / W=0.5[10,24]$.

Records of load vs. crack mouth opening displacements (CMOD) provide the experimental quantities to evaluate $J$. Using the measured plastic work defined by the plastic component of the area under the load versus CMOD curve, $A_{p l}^{C M O D}$, experimental fracture toughness values $\left(J_{c}\right)$ are obtained using the estimation procedure described by Kirk and Dodds [25]. Figure 4(a) reveals the pronounced effect of $a / W$ ratio and ductile grow th on $J_{c}$-values. Most of the specimens with $a / W=0.1$ experienced ductile crack extensions $(\Delta a)$ of $0.5-0.75 \mathrm{~mm}$ prior to cleavage fracture. In contrast, specimens with $a / W=0.5$ exhibited completely brittle behavior with no measurable crack extension prior to cleavage fracture. These results demonstrate the effect of severe constraint loss coupled with ductile tearing on the macroscopic fracture toughness, which leads to the increase in the $J_{c}$-values for the shallow crack specimens.

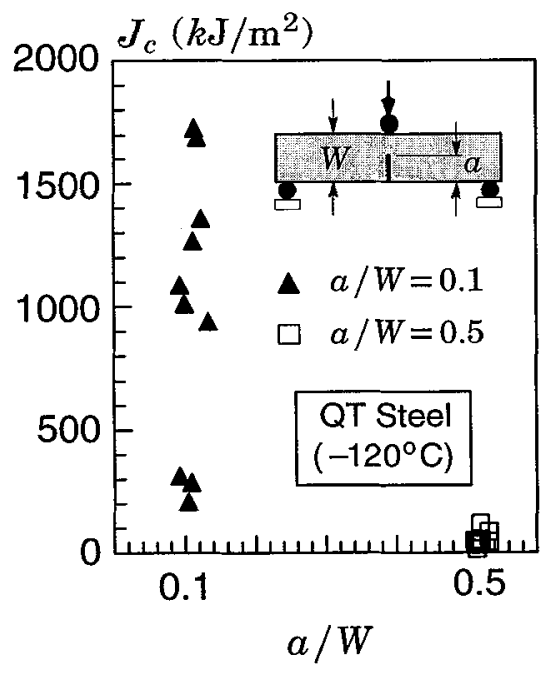

(a)

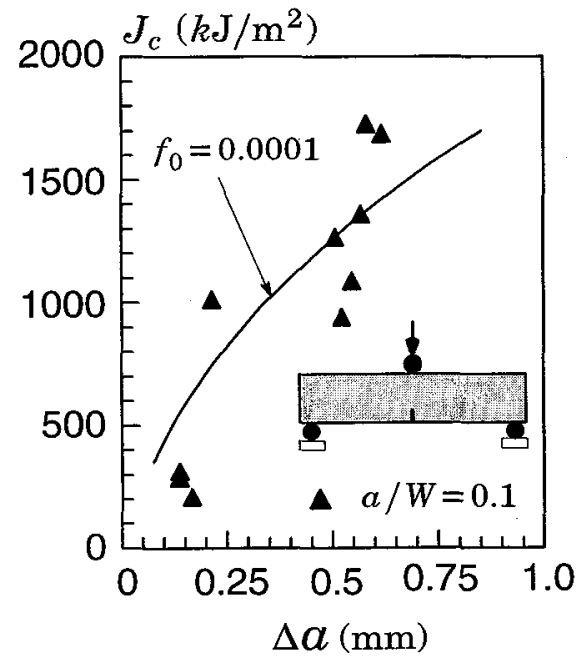

(b)

Figure 4 (a) Experimental cleavage fracture toughness data (SE(B) test results) for QT steel at $-120^{\circ} \mathrm{C}$. (b) Computed crack growth resistance curve for the SE(B) specimens with $a / W=0.1$.

\subsection{Prediction of $a / W$ and $\Delta a$ Effects on Toughness}

The procedure used here to predict the combined effects of $a / W$ ratio and ductile tearing for the experimental cleavage fracture toughness data follows a modified form of Dodds and Anderson toughness scaling model [16, 17] introduced by Ruggieri and Dodds [10]. Based upon micromechanics considerations, the modified scaling model requires the attainment of a specified value for the Weibull stress to trigger cleavage fracture in different specimens even though $J$-values may differ markedly. The notion of the Weibull stress as a crack-tip driving force establishes a function of the applied load and geometry which describes the local, crack-tip response for cleavage fracture. By postulating a critical value of the Weibull stress at fracture, the distribution of measured toughness values for one configuration may then be rationally employed to predict toughness distributions for other configurations. Here, we 
predict the distribution of cleavage fracture values for the shallow-crack specimens $(\alpha / W=0.1)$ using the distribution of measured fracture toughness for the $\mathrm{SE}(\mathrm{B})$ specimens with $a / W=0.5$.

The research code WSTRESS [22] is used to determine the material dependent value for the shape parameter, $m$, appearing in the Weibull stress as expressed by Eq. (2) and Eq. (3). Finite element solutions are generated using the WARP3D code [23] which: (1) implements the GT and Mises constitutive models in a finite-strain framework, (2) provides automatic cell extinction coupled to the GT model, and (3) evaluates the $J$-integral using a convenient domain integral procedure. The numerical procedure for estimating the shape parameter $m$ followed here is described fully in [9, 22]. The method, which avoids relying upon microscale measurements, employs the experimentally measured fracture toughness for the $\mathrm{SE}(\mathrm{B})$ specimens with $a / W=\mathbf{0 . 5}$. For the high-strength steel tested in these experiments, $m$ has the calibrated value of 24.9. Here, we note that this 3-D calibrated value of $m$ is larger than the calibrated value in plane-strain conditions $(m=15.6)$ obtained by $R \& D$ [10]. This aspect reflects differences in material sampling in the process zone ahead of the crack tip (3-D analysis versus plane-strain conditions) and the finer mesh employed in the present study. Nevertheless, the predicted distribution of cleavage fracture toughness for the $\mathrm{SE}(\mathrm{B})$ specimens with $a / W=0.1$ presented here does not differ significantly from that obtained by R\&D [10].

With the Weibull modulus $m$ known, only the parameters to model ductile growth in the finite element analyses for this material remain unspecified. The computational cell approach requires calibration of the the initial porosity, $f_{0}$, and cell size, $D$, from a measured set of $R$-curves. Ideally, $R$-curves obtained using $a / W=0.5$ specimens would be used for this procedure. However, deep-crack specimen $R$-curves are not available from the experimental investigation-at the test temperature, no ductile tearing occurs. Alternatively, experimental $R$-curves for other, low constraint geometries would be suitable to perform the calibration. Here, only the shallow-crack data is available and we use it to perform calibration of the computational cell parameters $\left(D, f_{0}\right)$.

Figure 4(b) shows the measured cleavage fracture data for the shallow-crack specimens plotted in an $R$-curve format. The cell with current porosity $f=0.1$ defines the current crack tip location, and thus $\Delta a$. This "operational" definition locates the crack tip in the region behind the peak stress where stresses decrease rapidly, but ahead of the very highly damaged region, where the GT model does not accurately predict material response. To improve the response of the GT model in low triaxiality regions along the crack front, we utilize the strain-controlled acceleration of void growth rate, Eq. (6) and Eq. (NO TAG), described in Section 3.2. The computed resistance curve shown on this figure is obtained using the values $D=200 \mu \mathrm{m}$ and $f_{0}=0.0001$ with $f_{E}=0.15$, and with nucleation parameters $\epsilon_{N}=1.0$, $S_{N}=0.05$ and $f_{N}=0.10$. This very low value for $f_{0}$ reflects the exceptionally fine microstructure of this material which produces the very high tearing resistance under sustained growth. Despite the relative scatter observed in the experimental (cleavage) values, the predicted $R$-curve captures the average evolution of crack growth. Since the primary interest lies in generating the accompanying near-tip stress fields for the growing crack, this calibrated model appears satisfactory.

Figure 5(a) shows the computed evolution of Weibull stress under increasing values of $J$ for the deep and shallow crack configurations. These curves provide the quantitative basis to predict the distribution of shallow-crack fracture toughness data from the measured distribution of deep-crack data. To illustrate the pronounced effect of ductile growth, Fig. 5(a) also includes the Weibull stress computed for the shallow-crack configuration neglecting growth. The Weibull stress for the no growth analysis attains a saturation value at $J /\left(b \sigma_{0}\right) \approx 0.025$, indicating that crack-tip stresses no longer increase. While both growth and no-growth curves for the shallow crack configuration display similar behavior at lower values of $J$, the stationary crack analysis clearly fails to predict the larger $J_{c}$-values measured in the experiments. As discussed earlier, the mesh employed in the growth analysis may not provide an adequate resolution of near-tip stress fields at low load levels. Thus, we adopt the following procedure to scale the distribution of measured fracture toughness for the $\mathrm{SE}(\mathrm{B})$ specimens with $a / W=0.5$ and predict the distribution of cleavage fracture values for the $S E(B)$ specimens with $a / W=0.1:$ a) the $\sigma_{w}$-curve for the no growth analysis is used until a saturation value occurs; b) the $\sigma_{w}$-curve for the growth analysis is used after ductile tearing occurs.

The Weibull stress values in these analyses derive from the current near-tip stress field (as provided by our definition of the instantaneous fracture process zone for the growing crack). We also compute the Weibull stress using the maximum stress that material elements in the process zone have experienced during the entire loading history (see Section 2.2). Figure 5(b) compares the evolution of $\sigma_{w}$ with $J$ for the shallow notch specimen (growth analysis) using both approaches. The results confirm our expectation that the Weibull stress values using this alternate treatment for material elements in 


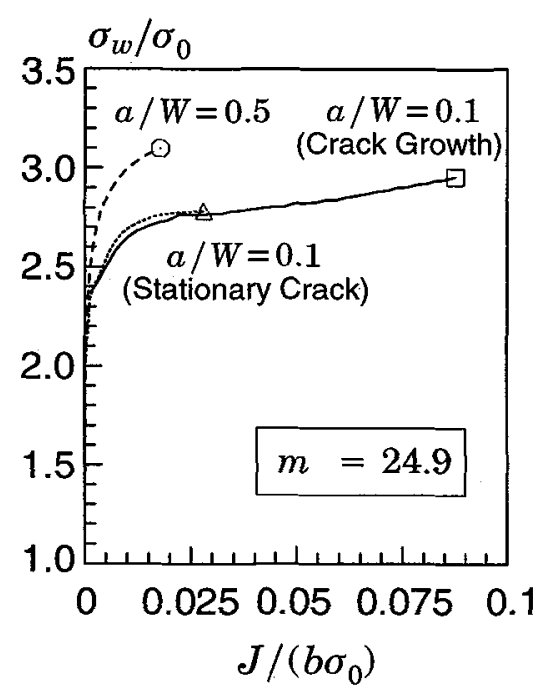

(a)

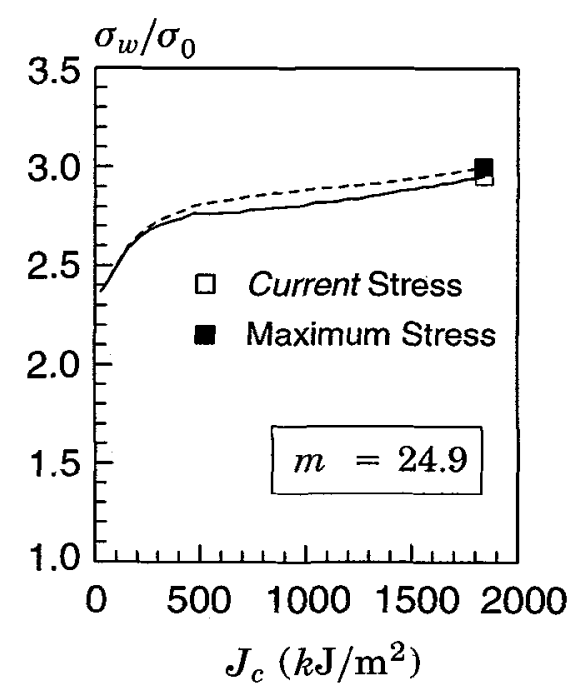

(b)

Figure 5 (a) Variation of the Weibull stress with increasing load for the SE(B) specimens. (b) Variation of the Weibull stress with increasing load for the $S E(B)$ specimens with $a / W=0.1$ using the current stress (instantaneous fracture process zone) and the maximum stress experienced by a material element.

the partially unloaded region ahead of the advancing tip should be similar. Clearly, it is the elevation of near-tip stress triaxiality and enlargement of the process zone ahead of the crack tip due to stable growth that plays the key role in increasing the total failure probability for the specimen.

The Weibull probability plot in Fig. 6 shows the predicted distributions of cleavage fracture toughness for the $\mathrm{SE}(\mathrm{B})$ specimen with $a / W=0.1$. The solid symbols in the plots indicate the experimental fracture toughness data for those specimens. Values of cumulative probability, $F$, are obtained by ordering the $J_{c}$-values and using $F=(i-0.5) / N$, where $i$ denotes the rank number and $N$ defines the total number of experimental toughness values. The solid line on that figure represents the predicted Weibull distribution generated from the distribution (not individual values from tested specimens) of the Weibull stress for the $\mathrm{SE}(\mathrm{B})$ specimen with $a / W=0.5$ using the procedure outlined by $R \& D$ [10, 22]. The dashed lines represent the $90 \%$ confidence bounds generated from the $90 \%$ confidence limits for the distribution of the Weibull stress for the $\mathrm{SE}(\mathrm{B})$ specimen with $a / W=0.5$. Here, we also note that the relatively flat region displayed at $J$-values $\approx 500 \sim 600 \mathrm{~kJ} / \mathrm{m}^{2}$ corresponds to the transition between the mesh for the no growth analysis and the one for the growth analysis. The predicted distribution displayed in Fig. 6 agrees well with the experimental data; further, most of the measured $J_{c}$-values lie within the $90 \%$ confidence bounds.

\section{Acknowledgements}

This investigation was supported by grants principally from the U.S. Nuclear Regulatory Commission, the Naval Surface Warfare Center - Annapolis Detachment (N61533-92-K-0030) and NASA-Ames (NCC2-5022). The authors acknowledge the useful discussions and contributions of Professors M. Toyoda and F. Minami (Osaka University) and Dr. Kim Wallin (VTT Finland). The authors also acknowledge Nippon Steel Corporation for making the CTOD test results available.

\section{References}

1. Beremin, F.M., Metall. Trans., Vol. 14A, pp. 2277-2287, 1983.

2. Gurson, A. L., J. Eng. Matls Tech., Vol. 99, pp. 2-15, 1977.

3. Tvergaard, V., Advances in Applied Mechanics, Vol. 27, pp. 83-151, 1990.

4. Batdorf, S. B. and Crose, J. G., J. Appl. Mech., Vol. 41, pp. 459-464, 1974. 


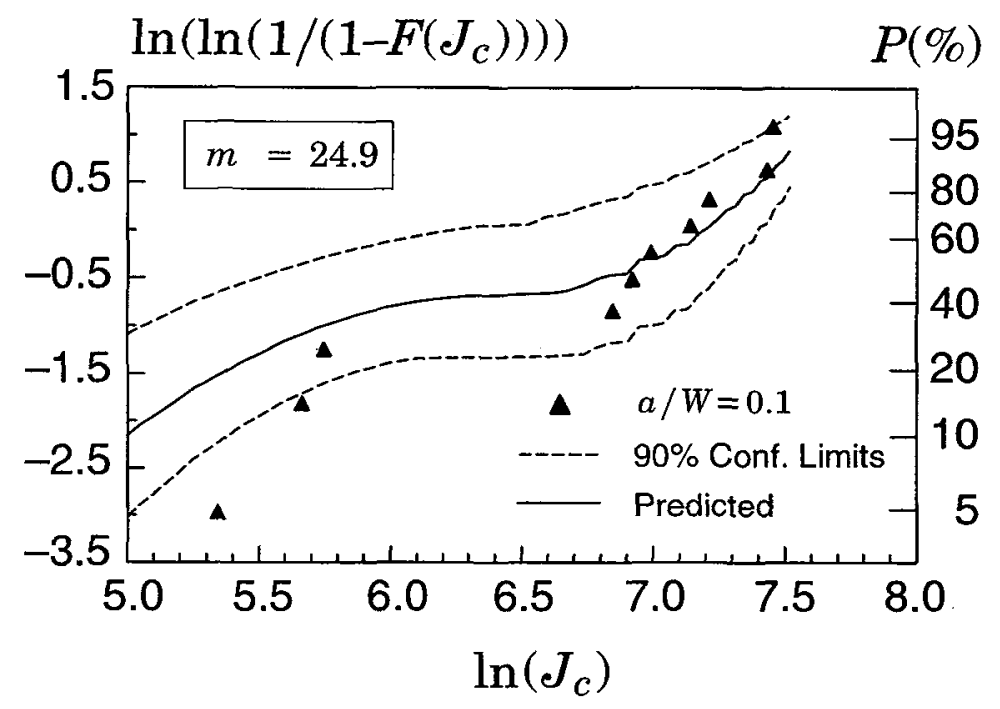

Figure 6 Prediction of the probability distribution for the cleavage fracture toughness of $\mathrm{SE}(\mathrm{B})$ specimens with $a / W=0.1$.

5. Wallin, K., Saario, T., and Torronen, K., Metal Sci., Vol. 18, pp. 13-16, 1984.

6. Brückner-Foit, A., Ehl, W., Munz, D. and Trolldenier, B., Fatigue Fract. Engng. Mater. Struct., Vol. 13, pp. 185-200, 1990.

7. Ruggieri, C., Minami, F. and Toyoda, M., J. Appl. Mech., Vol. 62, pp. 320-328, 1995.

8. Bakker, A. and Koers, R. W. J., "Prediction of Cleavage Fracture Events in the Brittle-Ductile Transition Region of a Ferritic Steel" in Defect Assessment in Components - Fundamentals and Applications, ESIS/EG9, Blauel and Schwalbe, Eds., Mechanical Engineering Publications, London, pp. 613-632, 1991.

9. Minami, F., Brückner-Foit, A, Munz, D.and Trolldenier, B., Int. J. Fracture, Vol. 54, pp. 197-210, 1992.

10. Ruggieri, C. and Dodds, R. H., "A Transferability Model for Brittle Fracture Including Constraint and Ductile Tearing Effects: A Probabilistic Approach”, to appear in Int. J. Fracture.

11. Mann, N. R., Schafer, R. E. and Singpurwalla, N. D., Methods for Statistical Analysis of Reliability and Life Data, Jonh Wiley \& Sons, New York, 1974.

12. Varias, A. G. and Shih, C. F., J. Mech. Phys. Solids, Vol. 41, pp. 835-861, 1993.

13. Dodds, R. H., Tang, M. and Anderson, T. L., "Effects of Prior Tearing on Cleavage Fracture Toughness in the Transition Region" in Constraint Effects in Fracture, Theory and Application, ASTM STP 1244, M. Kirk and A. Bakker, Eds., American Society for Testing and Materials, Philadelphia, Pennsylvania, 1993.

14. Zienkiewicz, O. C., The Finite Element Method, 3rd ed., McGraw-Hill, London, 1989.

15. Chu, C. C. and Needleman, A., J. Eng.. Matls. Tech., Vol. 102, pp. 249-256, 1980.

16. Dodds, R.H., Anderson, T.L., and Kirk, M.T., Int. J. Fracture, Vol. 48, pp. 1-22, 1991.

17. Anderson, T.L. and Dodds, R.H., J. Test. Eval., Vol. 19, pp. 123-134, 1991.

18. Xia, L. and Shih, C. F., J. Mech. Phys. Solids, Vol. 43, pp. 233-259, 1995.

19. Xia, L., Shih, C. F. and Hutchinson, J. W., J. Mech. Phys. Solids, Vol. 43, pp. 398-413, 1995.

20. Xia, L. and Shih, C. F., "Ductile Crack Growth - II. Void Nucleation and Geometry Effects on Macroscopic Fracture Behavior", to appear in J. Mech. Phys. Solids.

21. Xia, L. and Shih, C. F., "Ductile Crack Growth - III. Statistical Aspects of Cleavage Fracture After Tearing", to appear in J. Mech. Phys. Solids.

22. Ruggieri, C. and Dodds, R. H., "WSTRESS: Numerical Computation of Probabilistic Fracture Parameters". Structural Research Series (SRS) 608, ULLU-ENG-95-2013, University of Illinois at Urbana-Champaign, 1996.

23. Koppenhoefer, K., Gullerud, A., Ruggieri, C., Dodds, R. and Healy, B., "WARP3D: Dynamic Nonlinear Analysis of Solids Using a Preconditioned Conjugate Gradient Software Architecture", Structural Research Series (SRS) 596, UILUENG-94-2017, University of Illinois at Urbana-Champaign, 1994.

24. Toyoda, M., Minami, F., Matsuo, T., Hagiwara, Y and Inoue, T., "Effect of Work Hardening Properties of High Strength Steels on Cleavage/Ductile Fracture Resistance", Prep. National Meeting of the Japan Welding Society, Vol. 49, pp. 112-113, 1991 (in Japanese).

25. Kirk, M.T. and Dođds, R.H., J. Test. Evaluation, Vol. 24, No. 4, pp. 228-238, 1993. 